\title{
ARITHMETIC REPRESENTATIONS OF REAL NUMBERS IN TERMS OF SELF-SIMILAR SETS
}

\author{
Kan Jiang and Lifeng Xi \\ Ningbo University, Department of Mathematics \\ Ningbo 315211, P. R. China; jiangkan@nbu.edu.cn \\ Ningbo University, Department of Mathematics \\ Ningbo 315211, P. R. China; xilifeng@nbu.edu.cn
}

\begin{abstract}
Suppose $n \geq 2$ and $\mathcal{A}_{i} \subset\{0,1, \cdots,(n-1)\}$ for $i=1, \cdots, l$, let $K_{i}=\bigcup_{a \in \mathcal{A}_{i}} n^{-1}\left(K_{i}+\right.$ a) be self-similar sets contained in $[0,1]$. Given $m_{1}, \cdots, m_{l} \in \mathbf{Z}$ with $\prod_{i} m_{i} \neq 0$, we let

$$
S_{x}=\left\{\left(y_{1}, \cdots, y_{l}\right): m_{1} y_{1}+\cdots+m_{l} y_{l}=x \text { with } y_{i} \in K_{i} \forall i\right\} .
$$

In this paper, we analyze the Hausdorff dimension and Hausdorff measure of the following set

$$
U_{r}=\left\{x: \operatorname{Card}\left(S_{x}\right)=r\right\},
$$

where $\operatorname{Card}\left(S_{x}\right)$ denotes the cardinality of $S_{x}$, and $r \in \mathbf{N}^{+}$. We prove under the so-called covering condition that the Hausdorff dimension of $U_{1}$ can be calculated in terms of some matrix. Moreover, if $r \geq 2$, we also give some sufficient conditions such that the Hausdorff dimension of $U_{r}$ takes only finite values, and these values can be calculated explicitly. Furthermore, we come up with some sufficient conditions such that the dimensional Hausdorff measure of $U_{r}$ is infinity. Various examples are provided. Our results can be viewed as the exceptional results for the classical slicing problem in geometric measure theory.
\end{abstract}

\section{Introduction}

Representation of real numbers is a topic of great interest in number theory. There are many approaches which can represent real numbers, for instance, the $\beta$-expansions [18, 1, 28, 3, 6, 25], the continued fractions [11, 10], multiplication (division, quadratic sum) on fractal sets [31, 2], the Lüroth expansions [5], and so forth. These representations are related to many different mathematical aspects, for instance, the matrix theory, ergodic theory, fractal geometry, Diophantine approximation, combinatorics, and so on. Different representations have distinct properties from various aspects. Investigating the ergodic, fractal and combinatorial properties of these representations consists of one of the main directions in number theory.

Expansions in non-integer bases were pioneered by Rényi [25] and Parry [23]. Let $1<\beta<2$. Given any $x \in\left[0,(\beta-1)^{-1}\right]$, a sequence $\left(a_{n}\right) \in\{0,1\}^{\infty}$ is called a $\beta$ expansion of $x$ if $x=\sum_{n=1}^{\infty} a_{n} \beta^{-n}$. Sidorov [26] proved that given any $1<\beta<2$, then almost every point in $\left[0,(\beta-1)^{-1}\right]$ has uncountably many expansions. In fact, Sidorov [27], Dajani and de Vries [7] proved that given any $1<\beta<2$, then Lebesgue almost every point has uncountably many universal expansions. Erdôs et al. [13] proved that there exist some $1<\beta<2$ and $x \in\left[0,(\beta-1)^{-1}\right]$ such that $x$ has precisely $k$ different $\beta$-expansions. However, up to now, there are few results concerning with the

https://doi.org/10.5186/aasfm.2019.4463

2010 Mathematics Subject Classification: Primary 28A80.

Key words: Fractal, self-similar set, unique representation, section, projection.

Lifeng $\mathrm{Xi}$ is the corresponding author. The work is supported by National Natural Science Foundation of China (Nos. 11831007, 11771226, 11701302, 11371329, 11471124, 11671147). The work is also supported by K. C. Wong Magna Fund in Ningbo University. 
set of points with exactly $k$ different $\beta$-expansions, see $[9,8]$ and references therein. In particular, if $x$ has a unique expansion (such point is called the univoque point), then there are many results $[12,14,19]$. The main reason is that we may give an criterion of the unique expansions. As such we can discuss the set of points with unique expansions from the symbolic point of view.

Representation of real numbers also arises naturally from Diophantine approximation. Let $x$ be a real number. We say that $x$ is badly approximable if there exists a positive integer $n$ such that for any rational number $p / q$,

$$
|x-p / q|>\left(n q^{2}\right)^{-1} \text {. }
$$

Clearly, the set of badly approximable points is of Lebesgue measure zero. However, Hall [15] proved that every real number can be expressed as the sum of two badly approximable numbers. For any $m \geq 2$, let $F(m)$ denote the set of numbers

$$
F(m)=\left\{\left[t, a_{1}, a_{2}, \cdots\right]: t \in \mathbf{Z}, 1 \leq a_{i} \leq m \text { for } i \leq 1\right\},
$$

where

$$
\left[t, a_{1}, a_{2}, \cdots\right]=t+\frac{1}{a_{1}+\frac{1}{a_{2}+\cdots}} .
$$

Hall [15] proved that $F(4)+F(4)=\mathbf{R}$, where $A \pm B=\{x \pm y: x \in A, y \in B\}$. Astels [30] proved that

$$
F(2)+F(5)=\mathbf{R}, \quad F(2)-F(5)=\mathbf{R}, \quad F(3)-F(4)=\mathbf{R} .
$$

There are many other related results for the arithmetic sum of $F(m)$, see the references in $[15,30]$.

The above two representations are prevalent in number theory. In this paper we shall consider the so-called arithmetic representation of the real numbers. First, we introduce some background of this representation. Given two non-empty sets $A, B \subset \mathbf{R}$. Define $A * B=\{x * y: x \in A, y \in B\}$, where $*$ is,,$+- \times$ or $\div$ (when $*=\div, y \neq 0$ ). We call $a=x * y, x \in A, y \in B$ an arithmetic representation in terms of $A$ and $B$. Shortly, we may say $a=x * y$ is an arithmetic representation if there is no fear of ambiguity. It is well-known that for the middle-third Cantor set $C$,

$$
C-C=\{x-y: x, y \in C\}=[-1,1] .
$$

Therefore, for any $a \in[-1,1]$, there are some $x, y \in C$ such that $a=x-y$. The first proof of this result is due to Steinhaus [29] in 1917. Recently, Athreya, Reznick, and Tyson [2] considered the multiplication on the middle-third Cantor set, and proved that $17 / 21 \leq \mathcal{L}(C \cdot C) \leq 8 / 9$, where $\mathcal{L}$ denotes the Lebesgue measure. Moreover, they also proved that the division on $C$, denoted by $\frac{C}{C}$, is exactly the union of some closed sets. In [31], Tian et al. defined a class of overlapping self-similar sets as follows: let $K$ be the attractor of the IFS

$$
\left\{f_{1}(x)=\lambda x, f_{2}(x)=\lambda x+c-\lambda, f_{3}(x)=\lambda x+1-\lambda\right\},
$$

where $f_{1}(I) \cap f_{2}(I) \neq \emptyset,\left(f_{1}(I) \cup f_{2}(I)\right) \cap f_{3}(I)=\emptyset$, and $I=[0,1]$ is the convex hull of $K$. Then $K \cdot K=[0,1]$ if and only if $(1-\lambda)^{2} \leq c$. Equivalently, they gave a necessary and sufficient condition such that for any $x \in[0,1]$ there exist some $y, z \in K$ such that $x=y z$.

Motivated by the multiple $\beta$-expansions, generally it is natural to analyze the set of points in $A * B=\{x * y: x \in A, y \in B\}$ such that these points have exactly $r$ 
different representations, i.e. we want to analyze the following set

$$
U_{r}=\{x \in A * B: x \text { has exactly } r \text { arithmetic representations }\},
$$

where $r \in \mathbf{N}^{+}$. In this paper, we assume the above algorithm $*$ is + or - . For the classical middle-third Cantor set, take $1 / 3 \in C-C=[-1,1]$, it is not difficult to prove that $1 / 3$ has only three arithmetic representations in $C-C$, i.e.

$$
\frac{1}{3}=\frac{1}{3}-0=\frac{2}{3}-\frac{1}{3}=1-\frac{2}{3} \text {. }
$$

We may give a new explanation for Steinhaus' result from the projectional perspective. Note that $C-C$ is conjugated to $\sqrt{2} \operatorname{Proj}_{\theta}(C \times C)$, where $\theta=\frac{3 \pi}{4}$ and $\operatorname{Proj}_{\theta}$ denotes the orthogonal projection onto $L_{\theta}$ which is the line through the origin in direction $\theta$. Since $C-C=[-1,1]$, it follows that

$$
\operatorname{dim}_{H}\left(\operatorname{Proj}_{\theta}(C \times C)\right)=\min \left\{\operatorname{dim}_{H}(C)+\operatorname{dim}_{H}(C), 1\right\} .
$$

In other words, the orthogonal projection of $C \times C$ to the line $y=-x$ does not drop the expected dimension. Indeed, similar result is still correct for a general class of self-similar sets. Peres and Shmerkin [24], Hochman and Shmerkin [16] proved the following elegant result.

Let $K_{1}$ and $K_{2}$ be two self-similar sets with IFS's $\left\{f_{i}(x)=r_{i} x+a_{i}\right\}_{i=1}^{n}$ and $\left\{g_{j}(x)=r_{j}^{\prime} x+b_{j}\right\}_{j=1}^{m}$, respectively. If there are some $r_{i}, r_{j}^{\prime}$ such that

$$
\frac{\log \left|r_{i}\right|}{\log \left|r_{j}^{\prime}\right|} \notin \mathbf{Q},
$$

then

$$
\operatorname{dim}_{H}\left(K_{1}+K_{2}\right)=\min \left\{\operatorname{dim}_{H}\left(K_{1}\right)+\operatorname{dim}_{H}\left(K_{2}\right), 1\right\},
$$

and $\operatorname{dim}_{H}\left(K_{1}+K_{2}\right)=\operatorname{dim}_{P}\left(K_{1}+K_{2}\right)=\operatorname{dim}_{B}\left(K_{1}+K_{2}\right)$. The condition in the above result is called the irrationality condition. The result above indeed states that under the irrationality condition, the Hausdorff dimension of the projection of two self-similar sets through the angle $\pi / 4$ does not drop.

Now we go back to the middle-third Cantor set, and consider a slicing problem, i.e. given $t \in[-1,1]$, then the set $U_{r}$ in $(1.1)$, is

$$
U_{r}=\{t \in[-1,1]: \operatorname{Card}(\{y-x=t\} \cap(C \times C))=r\},
$$

where $r \in \mathbf{N}^{+}$. In other words, the multiple representational problem is indeed a slicing problem in geometric measure theory. In this paper, we shall consider the arithmetic addition or subtraction for more than two Cantor sets. First, we give some basic definitions.

Suppose $n \geq 2$ and $\mathcal{A}_{i} \subset\{0,1, \cdots,(n-1)\}$ for $i=1, \cdots, l$, let

$$
K_{i}=\bigcup_{a \in \mathcal{A}_{i}} \frac{K_{i}+a}{n}
$$

be self-similar sets contained in $[0,1]$. Fix $\mathbf{m}=\left(m_{1}, \cdots, m_{l}\right) \in \mathbf{Z}^{l}$ with $\prod_{i} m_{i} \neq 0$ denote

$$
S_{x}=\left\{\mathbf{y} \in \prod_{i=1}^{l} K_{i}:(\mathbf{m}, \mathbf{y})=m_{1} y_{1}+\cdots+m_{l} y_{l}=x\right\} .
$$

In this paper, we will focus on the fractal dimension of

$$
U_{r}=\left\{x: \operatorname{Card}\left(S_{x}\right)=r\right\} \text { for } r<\infty,
$$

It is worthwhile pointing out that if $\operatorname{Card}\left(S_{x}\right)=1$ then there is a unique solution for the equation

$$
x=m_{1} y_{1}+\cdots+m_{l} y_{l} \quad \text { with } y_{i} \in K_{i} \forall i
$$


Let $m_{*}=\sum_{m_{i}<0} m_{i}$ and $m^{*}=\sum_{m_{i}>0} m_{i}$ where $\sum_{b \in \emptyset} b=0$. Given a subset $B$ of $\mathbf{R}^{l}$, we write $(\mathbf{m}, B)=\bigcup_{b \in B}(\mathbf{m}, b)$. For $\mathbf{i}=\left(i_{1}, \cdots, i_{l}\right) \in \prod_{i=1}^{l} \mathcal{A}_{i}$ and we write the small cube $\mathbf{c}_{\mathbf{i}}=\frac{\left(i_{1}, \cdots, i_{l}\right)+[0,1]^{l}}{n}$. We say that the covering condition holds for $\prod_{i=1}^{l} K_{i}$ with respect to $\mathbf{m}$, if

$$
\bigcup_{\mathbf{i} \in \prod_{i=1}^{l} \mathcal{A}_{i}}\left(\mathbf{m}, \mathbf{c}_{\mathbf{i}}\right)=\left(\mathbf{m},[0,1]^{l}\right)
$$

where $\left(\mathbf{m},[0,1]^{l}\right)=\left[m_{*}, m^{*}\right]$ and $\left(\mathbf{m}, \mathbf{c}_{\mathbf{i}}\right)=n^{-1}\left((\mathbf{m}, \mathbf{i})+\left[m_{*}, m^{*}\right]\right)$.

We call $I=n^{-1}[u, u+1] \subset\left[m_{*}, m^{*}\right]$ with $u \in \mathbf{Z}$ an integer interval, and $\left\{J_{t}=\right.$ $[t, t+1]\}_{t \in\left[m_{*}, m^{*}\right) \cap \mathbf{Z}}$ working intervals. We say $I$ is of type $t$ (with respect to the small cube $\left.c_{\mathbf{i}}\right)$, if $u-(\mathbf{m}, \mathbf{i})=t \in\left[m_{*}, m^{*}-1\right] \cap \mathbf{Z}$ for some $\mathbf{i} \in \prod_{i=1}^{l} \mathcal{A}_{i}$, i.e., $t$ is the relative position of $I$ according to the projection interval $\left(\mathbf{m}, \mathbf{c}_{\mathbf{i}}\right)=n^{-1}\left((\mathbf{m}, \mathbf{i})+\left[m_{*}, m^{*}\right]\right)$ of the small cube $\mathbf{c}_{\mathbf{i}}$. For $t \in\left[m_{*}, m^{*}\right) \cap \mathbf{Z}$, the corresponding geometric type is $[0,1]^{l} \cap\left\{\mathbf{y}:(\mathbf{m}, \mathbf{y}) \in J_{t}\right\}$ or its similar copy. Two integer intervals $I_{1}=n^{-1}\left[u_{1}, u_{1}+1\right]$ and $I_{2}=n^{-1}\left[u_{2}, u_{2}+1\right]$ are said to be congruent modulo $n$, if $u_{1} \equiv u_{2}(\bmod n)$, i.e., $I_{2}=I_{1}+k$ for some $k \in \mathbf{Z}$.

For a directed graph, we give a partial order on its strongly connected components $\left\{H_{i}\right\}_{i}$, we denote $H \prec H^{\prime}$, if $H=H^{\prime}$ or there is a directed path from one vertex of $H$ to another vertex of $H^{\prime}$. Let $\rho\left(H_{i}\right)$ be the spectral radius of the matrix with respect to the subgraph restricted in $H_{i}$. We say an infinite sequence $v_{i_{1}} v_{i_{2}} \cdots v_{i_{k}} v_{i_{k+1}} \cdots$ of vertexes is admissible if there is a directed edge from $v_{i_{k}}$ to $v_{i_{k+1}}$ for all $k$.

In this paper, we need three directed graphs.

(1) The first graph has the vertex set of all integer intervals. For two vertexes (or integer intervals) $I_{1}$ and $I_{2}$, there is a directed edge from $I_{1}$ to $I_{2}$, denoted by $I_{1} \rightarrow I_{2}$, if and only if there exists an integer $t \in\left[m_{*}, m^{*}-1\right]$ such that $I_{1}$ is of type $t$ and $I_{2} \subset J_{t}$. We denote $I \longmapsto J_{t}$ if the integer interval $I$ is of type $t$.

(2) The second graph is a subgraph of the first. Let $\Xi$ denote the collection of integer intervals $I$ such that there is a unique $\mathbf{i} \in \prod_{i=1}^{l} \mathcal{A}_{i}$ satisfying $I \subset\left(\mathbf{m}, \mathbf{c}_{\mathbf{i}}\right)$. Then we obtain a directed subgraph $G_{\Xi}$ of the first one. From this directed graph, we obtain a 0-1 transition matrix $M$ with its spectral radius $\rho(M)$. Denote by $\left\{\Xi_{i}\right\}_{i}$ the strongly connected components of $G_{\Xi}$. In the graph $G_{\Xi}$, we say that a strongly connected component $\Xi_{i}$ can reach $J_{t}$, if there is a directed path in $G_{\Xi}$ from one vertex of $\Xi_{i}$ to another vertex of type $t$. We also say that $\left[m_{*}, m^{*}\right]$ is dominated by $\Xi$ for $d>0$, if each $J_{t}$ can be reached by some $\Xi_{i}$ with $\frac{\log \rho\left(M_{i}\right)}{\log n} \geq d$.

(3) The third graph $G^{*}$ contains $G_{\Xi}$. A subset $\omega$ of $\Xi$ is said to be congruent, if any two of $\omega$ are congruent. For congruent subset $\omega$, let

$$
\mathcal{D}(\omega)=\left\{t \text { : there is an element of } \omega \text { contained in } J_{t}=[t, t+1]\right\} .
$$

Let the vertex set of $G^{*}$ be the collection of all congruent subsets of $\Xi$. Then there is a directed edge from $\omega$ to $\omega^{\prime}$, if and only if for any $I \in \omega$, there exists some $I^{\prime} \in \omega^{\prime}$ such that $I \rightarrow I^{\prime}$ (in the first graph), and for any $I^{\prime} \in \omega^{\prime}$, there exists some $I \in \omega$ such that $I \rightarrow I^{\prime}$ (in the first graph). Denote by $\left\{\Omega_{j}\right\}_{j}$ the strongly connected components of $G^{*}$. Note that $G_{\Xi}$ is a subgraph of $G^{*}$, and any strongly connected component of $G_{\Xi}$ is also a strongly connected component of $G^{*}$, hence $\left\{\rho\left(\Xi_{i}\right)\right\}_{i} \subset\left\{\rho\left(\Omega_{j}\right)\right\}_{j}$.

Theorem 1. Let $s=\frac{\log \rho(M)}{\log n}$. Then we have the following results.

(1) $\operatorname{dim}_{H} U_{1} \geq s$ and $\mathcal{H}^{s}\left(U_{1}\right)>0$. 
(2) Suppose the covering condition holds for $\prod_{i=1}^{l} K_{i}$ with respect to $\mathbf{m}$, then

$$
\operatorname{dim}_{H} U_{1}=s \text {. }
$$

(3) Suppose the covering condition holds for $\prod_{i=1}^{l} K_{i}$ with respect to $\mathbf{m}$ and $K_{i}$ satisfies the strong separation condition holds (for instance, $1 \notin \mathcal{A}_{i}-\mathcal{A}_{i}$ implies the strong separation condition) for all $i$. Suppose $s>0$, then

$$
\mathcal{H}^{s}\left(U_{1}\right)=\infty
$$

if and only if there are two different strongly connected components $\Xi_{i}$ and $\Xi_{j}$ in $G_{\Xi}$ such that $\rho\left(\Xi_{i}\right)=\rho\left(\Xi_{j}\right)=\rho(M)$ and $\Xi_{i} \prec \Xi_{j}$. In particular if $M$ is irreducible then

$$
0<\mathcal{H}^{s}\left(U_{1}\right)<\infty
$$

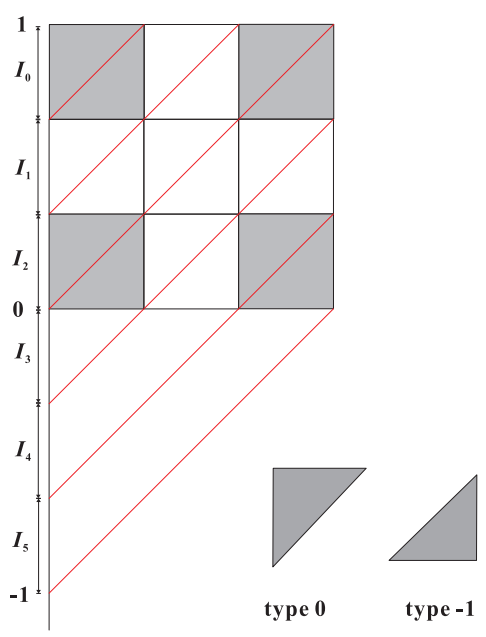

(a)

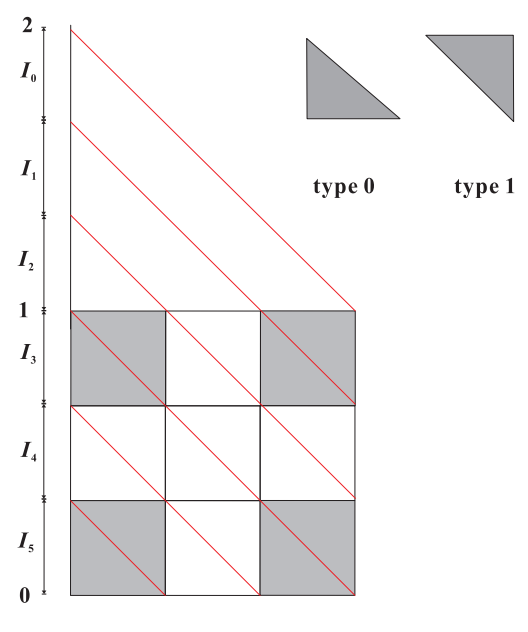

(b)

Figure 1. Example 1.

Example 1. As in part (a) of Fig. 1, let us consider the equation $x=-b_{1}+b_{2}$ with $b_{i} \in C$, where $C$ is the middle-third Cantor set and $\left(m_{1}, m_{2}\right)=(-1,1)$ with $m_{*}=-1$ and $m^{*}=1$. Now, the covering condition and the strong separation condition hold. Using the lines $\bigcup_{d \in 3^{-1}\{-3, \cdots, 3\}}\{(x, y): y-x=d\}$, we obtain six integer intervals $I_{0}, \cdots, I_{5}$ and working intervals $[0,1]$ and $[-1,0]$. Four small squares $\mathbf{c}_{(0,0)}, \mathbf{c}_{(0,2)}, \mathbf{c}_{(2,0)}$ and $\mathbf{c}_{(2,2)}$ are shown in part (a) of Fig. 1 . We have $\Xi=\left\{I_{0}, I_{1}, I_{4}, I_{5}\right\}$ and the transition matrix

$$
M=\left(\begin{array}{llll}
1 & 1 & 0 & 0 \\
0 & 0 & 1 & 1 \\
1 & 1 & 0 & 0 \\
0 & 0 & 1 & 1
\end{array}\right) \quad \text { with } \rho(M)=2
$$

then $\operatorname{dim}_{H} U_{1}=\frac{\log 2}{\log 3}=s$ and $0<\mathcal{H}^{s}\left(U_{1}\right)<\infty$ since $M$ is irreducible. In this example, $\operatorname{dim}_{H} U_{1}=\operatorname{dim}_{H} C=\frac{\log 2}{\log 3}$.

For part (b) of Fig. 1, let us consider the equation $x=b_{1}+b_{2}$ with $b_{i} \in C$, where $C$ is the middle-third Cantor set and $\left(m_{1}, m_{2}\right)=(1,1)$ with $m_{*}=0$ and $m^{*}=2$. We also have $\Xi=\left\{I_{0}, I_{1}, I_{4}, I_{5}\right\}$ and the same transition matrix $M$ with $\rho(M)=2$. Hence in this case $\operatorname{dim}_{H} U_{1}=\operatorname{dim}_{H} C=\frac{\log 2}{\log 3}$.

In the following examples, we have $\operatorname{dim}_{H} U_{1}<\min _{i}\left(\operatorname{dim}_{H} K_{i}\right)$ or $\operatorname{dim}_{H} U_{1}>$ $\max _{i}\left(\operatorname{dim}_{H} K_{i}\right)$. 


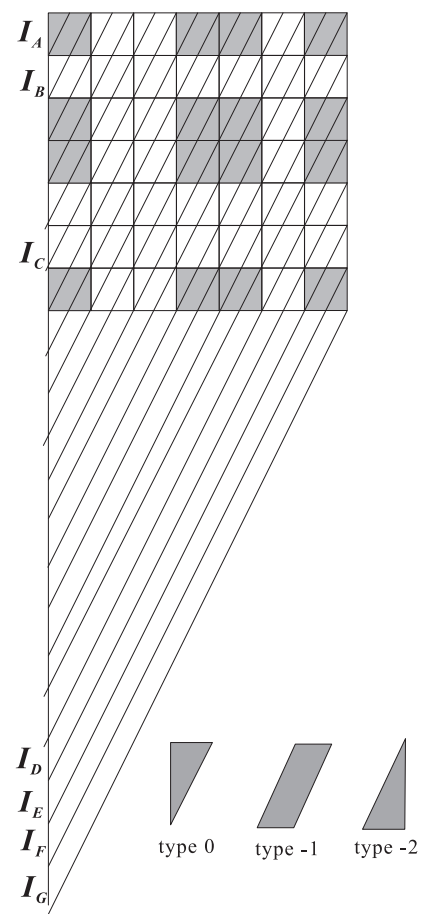

(a)

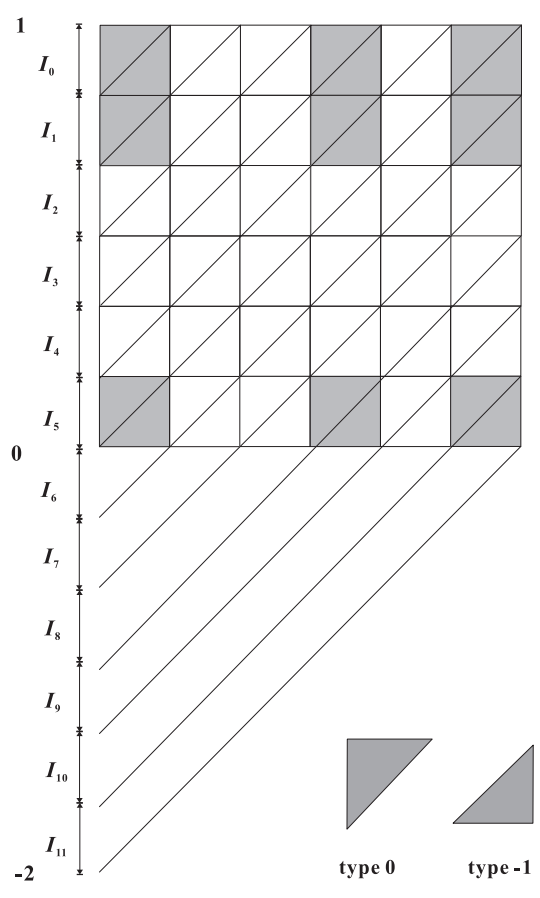

(b)

Figure 2. Examples 2 and 3.

Example 2. As in part (a) of Fig. 2, let us consider the equation $x=-2 b_{1}+b_{2}$ with $b_{i} \in K=\bigcup_{a \in\{0,3,4,6\}} \frac{K+a}{7}$, where $\left(m_{1}, m_{2}\right)=(-2,1)$ with $m_{*}=-2$ and $m^{*}=$ 1. The covering condition holds. We have $\Xi=\left\{I_{A}, I_{B}, I_{C}, I_{D}, I_{E}, I_{F}, I_{G}\right\}$ and the transition matrix

$$
M=\left(\begin{array}{lllllll}
1 & 1 & 1 & 0 & 0 & 0 & 0 \\
0 & 0 & 0 & 0 & 0 & 0 & 0 \\
0 & 0 & 0 & 1 & 1 & 1 & 1 \\
0 & 0 & 0 & 1 & 1 & 1 & 1 \\
1 & 1 & 1 & 0 & 0 & 0 & 0 \\
0 & 0 & 0 & 0 & 0 & 0 & 0 \\
0 & 0 & 0 & 1 & 1 & 1 & 1
\end{array}\right) \quad \text { with } \rho(M)=\frac{\sqrt{5}+3}{2}
$$

Then $\operatorname{dim}_{H} U_{1}=\frac{\log \frac{\sqrt{5}+3}{2}}{\log 7}=s$. In this example, the spectral radius is not an integer and $\operatorname{dim}_{H} U_{1}<\operatorname{dim}_{H} K=\frac{\log 4}{\log 7}$.

Example 3. As in part (b) of Fig. 2, let us consider the equation $x=-b_{1}+b_{2}$ with $b_{1} \in K_{1}=\bigcup_{a \in\{0,3,5\}} \frac{K_{1}+a}{6}$ and $b_{2} \in K_{2}=\bigcup_{a \in\{0,4,5\}} \frac{K_{2}+a}{6}$, where $\left(m_{1}, m_{2}\right)=$ $(-1,1)$ with $m_{*}=-1$ and $m^{*}=1$. The covering condition and the strong separation condition hold. We have $\Xi=\left\{I_{0}, I_{2}, I_{3}, I_{7}, I_{8}, I_{9}, I_{10}, I_{11}\right\}$ and the transition matrix

$$
M=\left(\begin{array}{llllllll}
1 & 1 & 1 & 0 & 0 & 0 & 0 & 0 \\
0 & 0 & 0 & 1 & 1 & 1 & 1 & 1 \\
1 & 1 & 1 & 0 & 0 & 0 & 0 & 0 \\
0 & 0 & 0 & 1 & 1 & 1 & 1 & 1 \\
1 & 1 & 1 & 0 & 0 & 0 & 0 & 0 \\
0 & 0 & 0 & 1 & 1 & 1 & 1 & 1 \\
1 & 1 & 1 & 0 & 0 & 0 & 0 & 0 \\
0 & 0 & 0 & 1 & 1 & 1 & 1 & 1
\end{array}\right) \quad \text { with } \rho(M)=4
$$


Then $\operatorname{dim}_{H} U_{1}=\frac{\log 4}{\log 6}=s$ and $0<\mathcal{H}^{s}\left(U_{1}\right)<\infty$ since $M$ is irreducible. Notice that in this example $\operatorname{dim}_{H} U_{1}>\max \left\{\operatorname{dim}_{H} K_{1}, \operatorname{dim}_{H} K_{2}\right\}=\frac{\log 3}{\log 6}$.

Given $j \in\{0, \cdots,(n-1)\}$, a $\|\mathbf{m}\|_{1} \times\|\mathbf{m}\|_{1}$ matrix $T_{j}=\left(b_{u v}\right)_{m_{*} \leq u, v \leq m^{*}-1}$ is defined by

$$
b_{u v}=\operatorname{Card}\left\{\mathbf{i} \in \prod_{i=1}^{l} \mathcal{A}_{i}:(n u+j)-(\mathbf{m}, \mathbf{i})=v\right\} .
$$

In fact, $b_{u v}>0$ if and only if $I_{(u, j)} \longmapsto J_{v}$, where $I_{(u, j)}=n^{-1}[n u+j, n u+j+1]$ and $J_{v}=[v, v+1]$. We also note that $b_{u v}$ is the number of small cubes $\mathbf{c}_{\mathbf{i}}$ such that $I_{(u, j)}$ has the relative position $v$ according to the projection interval $(\mathbf{m}, \mathbf{i})$ of the small cube $\mathbf{c}_{\mathbf{i}}$. Please see the following two examples 4 and 5 for this definition.

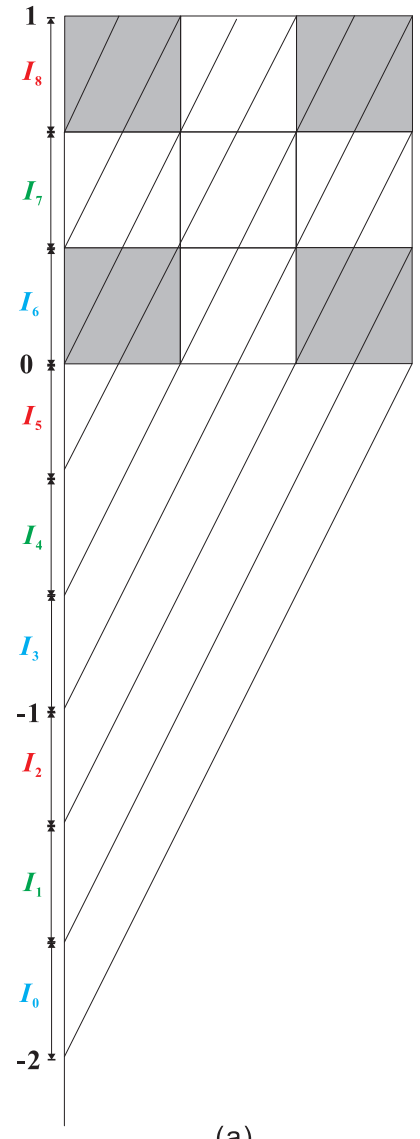

(a)

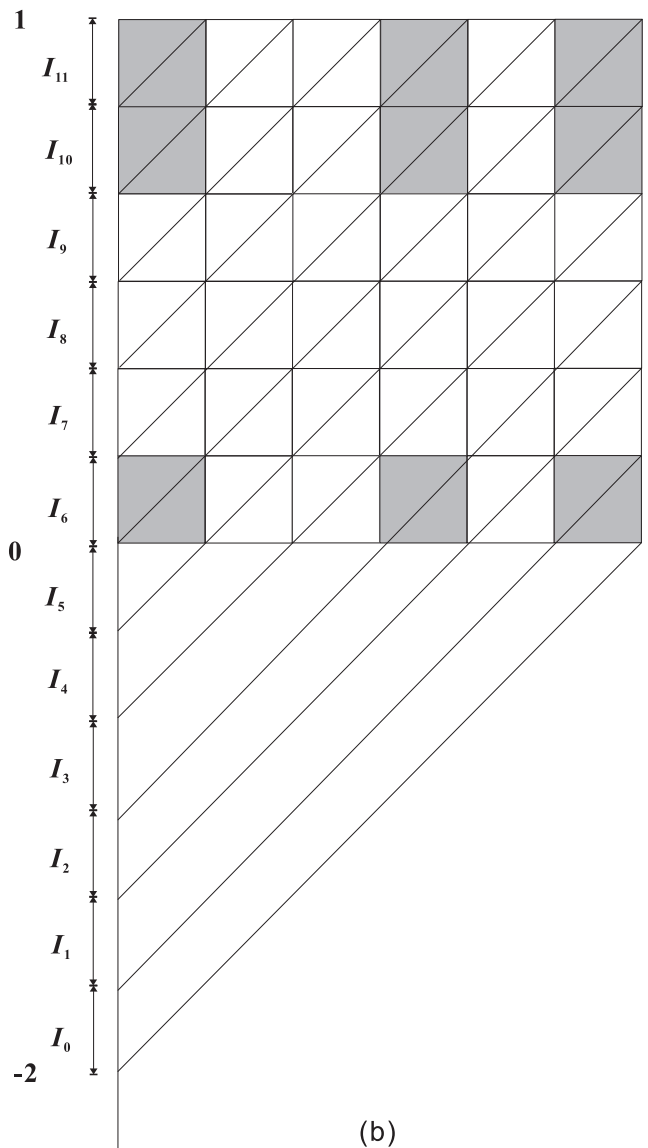

(b)

Figure 3. Examples 4 and 5.

Example 4. Consider $-2 C+C$ as in part (a) of Fig. 3. We have

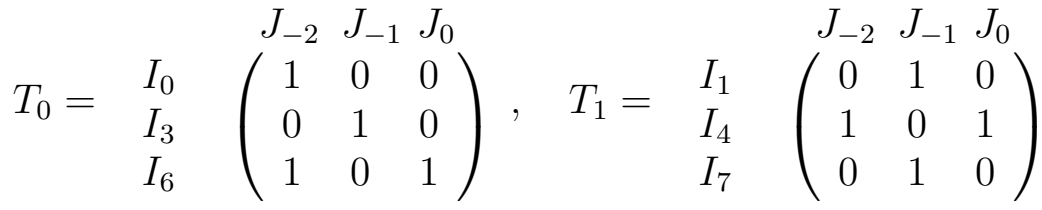

$$
\begin{aligned}
& \begin{array}{lll}
J_{-2} & J_{-1} & J_{0}
\end{array} \\
& \text { and } T_{2}=\begin{array}{c}
I_{2} \\
I_{5} \\
I_{8}
\end{array} \quad\left(\begin{array}{ccc}
1 & 0 & 1 \\
0 & 1 & 0 \\
0 & 0 & 1
\end{array}\right) \text {. }
\end{aligned}
$$


Example 5. Consider $-K_{1}+K_{2}$ for $K_{1}=\bigcup_{a \in\{0,3,5\}} \frac{K_{1}+a}{6}$ and $K_{2}=\cup_{a \in\{0,4,5\}} \frac{K_{2}+a}{6}$ as in part (b) of Fig. 3. We have

$$
\begin{aligned}
& J_{-1} J_{0} \\
& T_{0}=\begin{array}{l}
I_{0} \\
I_{6}
\end{array}\left(\begin{array}{ll}
1 & 0 \\
1 & 2
\end{array}\right), \quad T_{1}=\begin{array}{l}
I_{1} \\
I_{7}
\end{array}\left(\begin{array}{ll}
0 & 1 \\
1 & 1
\end{array}\right), \\
& J_{-1} J_{0} \quad J_{-1} J_{0} \\
& T_{2}=\begin{array}{l}
I_{2} \\
I_{8}
\end{array}\left(\begin{array}{ll}
1 & 0 \\
0 & 1
\end{array}\right), \quad T_{3}=\begin{array}{l}
I_{3} \\
I_{9}
\end{array}\left(\begin{array}{ll}
0 & 1 \\
1 & 0
\end{array}\right), \\
& J_{-1} J_{0} \quad J_{-1} J_{0} \\
& T_{4}=\begin{array}{ll}
I_{4} \\
I_{10}
\end{array}\left(\begin{array}{ll}
1 & 0 \\
1 & 1
\end{array}\right), \quad T_{5}=\begin{array}{ll}
I_{5} \\
I_{11}
\end{array}\left(\begin{array}{ll}
2 & 1 \\
0 & 1
\end{array}\right) .
\end{aligned}
$$

In fact, under the covering condition, by the method of [20], we can obtain that for Lebesgue almost all $x \in\left[m_{*}, m^{*}\right.$,

$$
\operatorname{dim}_{H} S_{x}=\operatorname{dim}_{B} S_{x}=\frac{\xi}{\log n},
$$

where $\xi$ is the Lyapunov exponent for the symmetric independent random product of $T_{0}, \cdots, T_{n-1}$, i.e.,

$$
\xi=\lim _{k \rightarrow \infty} \frac{\log \left\|T_{x_{1}} T_{x_{2}} \cdots T_{x_{k}}\right\|}{k}
$$

with $x_{k}$ i.i.d. random variables assuming the values $\{0,1, \cdots,(n-1)\}$ with equal probabilities. Related results can be found in $[4,21]$. When the direction is fixed, some general result on sections of self-similar sets can be found in [32].

A mapping $\psi: \bigcup_{I \in \Xi} \operatorname{int}(I) \rightarrow\left(m_{*}, m^{*}\right)$ is defined by

$$
\psi(x)=n\left(x-a_{I}\right)+t(I) \text { for all } x \in \operatorname{int}(I)=\left(a_{I}, a_{I}+n^{-1}\right),
$$

where $t(I)$ is the type of $I$. Then $\left.\psi\right|_{\operatorname{int}(I)}$ is a linear surjection from $I$ to $\operatorname{int}\left(J_{t}\right)=$ $(t, t+1)$ with factor $n$. Notice that if $x_{1}, x_{2} \in \bigcup_{I \in \Xi} \operatorname{int}(I)$ and $x_{1}=x_{2}+k$ with $k \in \mathbf{Z}$ and $x_{i} \in \operatorname{int}\left(I_{i}\right)$ for $i=1,2$, then

$$
\psi\left(x_{1}\right)-\psi\left(x_{2}\right)=n\left(k-a_{I_{1}}+a_{I_{2}}\right)+\left(t\left(I_{1}\right)-t\left(I_{2}\right)\right) \in \mathbf{Z} .
$$

That means the integer intervals containing $\psi\left(x_{1}\right)$ and $\psi\left(x_{2}\right)$ are congruent (modulo $n$ ) if $x_{1}, x_{2}$ lie in the interiors of the corresponding integer intervals respectively. In fact, the third graph defined above is based on this observation. Given $z \in$ $[0,1) \backslash\left\{\frac{q_{1}}{n^{q_{2}}}\right\}_{q_{1}, q_{2} \in \mathbf{Z}}$ and a subset $\mathcal{D}$ of $\mathbf{Z} \cap\left[m_{*}, m^{*}-1\right]$, we say that the vector $(z+p)_{p \in \mathcal{D}}$ (with index lying in $\mathbf{Z} \cap\left[m_{*}, m^{*}-1\right]$ ) has an infinite coding $\omega_{0} \omega_{1} \cdots \omega_{k} \cdots$ in $G^{*}$, if $\psi^{i}(z+p)$ belongs to the interior of some integer interval of $\Xi$ for each $i \geq 0$ and $p \in \mathcal{D}$, and $\omega_{i}$ is the smallest congruent subset of $\Xi$ containing $\bigcup_{p \in \mathcal{D}}\left\{\psi^{i}(z+p)\right\}$ for each $i \geq 0$ such that $\omega_{0} \omega_{1} \cdots \omega_{k} \cdots$ is admissible in $G^{*}$. Let $e_{i}$ be the $i$-th one of the natural basis on $\mathbf{R}^{\|\mathbf{m}\|_{1}}$ for $m_{*} \leq i \leq m^{*}-1$.

Theorem 2. Suppose the covering condition holds and $K_{i}$ satisfies the strong separation condition for each $i$. Then

$$
U_{r} \backslash\left\{\frac{q_{1}}{n^{q_{2}}}\right\}_{q_{1}, q_{2} \in \mathbf{Z}} \neq \emptyset
$$

if and only if there exist $i, j_{1}, \cdots, j_{k}$ such that

$$
r=\left\|e_{i} T_{j_{1}} \cdots T_{j_{k}}\right\|_{1}
$$


with $e_{i} T_{j_{1}} \cdots T_{j_{k}}=\left(\beta_{m_{*}}, \cdots, \beta_{m^{*}-1}\right)$ and $\mathcal{D}=\left\{p: \beta_{p} \neq 0\right\}$ satisfying

$$
\left\{z \in[0,1) \backslash\left\{\frac{q_{1}}{n^{q_{2}}}\right\}_{q_{1}, q_{2} \in \mathbf{Z}}:(z+p)_{p \in \mathcal{D}} \text { has an infinite coding in } G^{*}\right\} \neq \emptyset .
$$

The condition (1.3) implies that the set $\left\{\bar{\omega}=\omega_{i_{0}} \omega_{i_{1}} \cdots \omega_{i_{k}} \cdots\right.$ : the infinite sequence $\bar{\omega}$ is admissible in $G^{*}$ with $\mathcal{D}\left(\omega_{i_{0}}\right)=\mathcal{D}$ \} is non-empty. If the latter set is uncountable, then (1.3) follows. In particular, if $\mathcal{D}\left(\omega_{i_{0}}\right)=\mathcal{D}$ and $\omega_{i_{0}} \in \Omega_{i}$ such that $\rho\left(\Omega_{j}\right)>0$ with $\Omega_{i} \prec \Omega_{j}$ for some $\Omega_{j}$, then (1.3) follows.

Theorem 3. Suppose the covering condition holds. Let $U_{r} \neq \emptyset$ with $r \geq 2$ and

$$
d_{r}=\operatorname{dim}_{H} U_{r}
$$

(1) Then we have

$$
d_{r} \leq \frac{\log \rho(M)}{\log n}
$$

(2) Moreover, we assume that $K_{i}$ satisfies the strong separation condition for each $i$, then either $U_{r}$ is countable, or

$$
d_{r} \in\left(\bigcup_{i} \max \left\{\frac{\log \rho\left(\Omega_{j}\right)}{\log n}: \Omega_{i} \prec \Omega_{j}\right\}\right) \backslash\{0\} \text { and } \mathcal{H}^{d_{r}}\left(U_{r}\right)>0 \text {. }
$$

(3) Suppose $K_{i}$ satisfies the strong separation condition for all $i$ and $\left[m_{*}, m^{*}\right]$ is dominated by $\Xi$ for $d_{r}$, then $\mathcal{H}^{d_{r}}\left(U_{r}\right)=\infty$. In particular, if $M$ is irreducible and the elements in $\Xi$ can reach every working interval, then

$$
\mathcal{H}^{d_{r}}\left(U_{r}\right)=\infty
$$

Example 6. Let us consider $C-C$ again. We note that for $\omega=\left\{I_{0}, I_{4}\right\}$ we only find the edges from $\omega$ to $\left\{I_{0}\right\}$ and $\left\{I_{1}\right\}$, and the edges from $\left\{I_{1}, I_{5}\right\}$ to $\left\{I_{0}\right\}$ and $\left\{I_{1}\right\}$. The effective part of the matrix is the submatrix with respect to $G_{\Xi}$. Then the non-empty set $U_{r}$ is countable or has dimension $\log 2 / \log 3$. We also check that $M$ in Example 1 is irreducible and the elements in $\Xi$ can reach every working interval. By Theorem $3, U_{r}$ is countable or $\operatorname{dim}_{H} U_{r}=\log 2 / \log 3$ with infinite Hausdorff measure.

Now, we will find $r$ such that $U_{r}$ is non-empty. Ignoring a countable set $\left\{\frac{q}{3^{p}}\right\}_{p, q \in \mathbf{Z}}$, we can take the infinite coding with digit $\{0,1,2,3,4,5\}$ to represent the number in $[-1,1]$ uniquely, where digit $j$ represents $I_{j}$. Now, the coding is not free, we have the following rules:

(1) If the current digit is 0,2 or 4 , then the next digit shall be taken in $\{0,1,2\}$;

(2) If the current digit is 1,3 or 5 , then the next digit shall be taken in $\{3,4,5\}$. Let

$$
\eta(i)= \begin{cases}1 & \text { if } i=0,1,4 \text { or } 5 \\ 2 & \text { otherwise }\end{cases}
$$

Then

$$
\operatorname{Card}\left\{\left(b_{1}, b_{2}\right) \in C \times C: x=-b_{1}+b_{2}\right\}=\prod_{k} \eta\left(i_{k}\right),
$$

if $x$ has coding $i_{0} i_{1} i_{2} \cdots i_{k} \cdots$, when we ignore a countable set $\left\{\frac{q_{1}}{3^{q_{2}}}\right\}_{q_{1}, q_{2} \in \mathbf{Z}}$. 

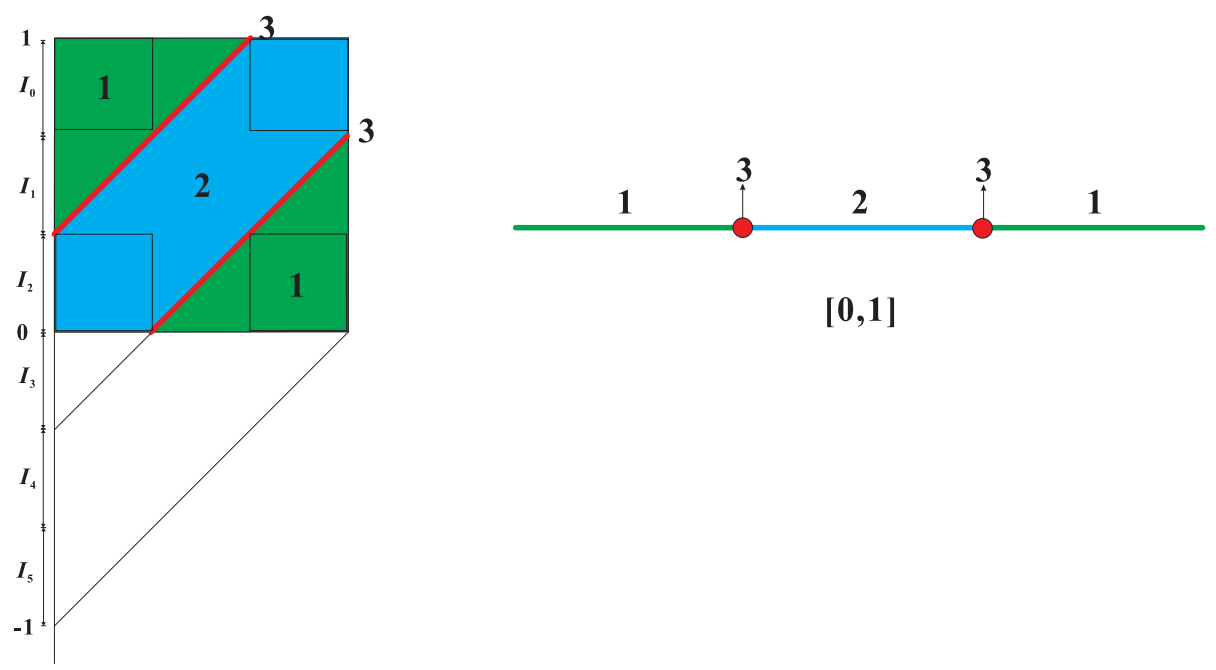

$[0,1]$

Figure 4. Example 6.

Let $P:\{0,1,2,3,4,5\} \rightarrow\{0,1,2\}$ be a projection defined by $P(2 j)=P(2 j+1)=$ $j$, we simplify this above model to the unit interval with 3 -adic expansion and take

$$
\bar{\eta}(j)= \begin{cases}1 & \text { if } j=0,2, \\ 2 & \text { if } j=1 .\end{cases}
$$

Then the cardinality is $\prod_{k} \bar{\eta}\left(j_{k}\right)$ for the 3 -adic expansion $0 . j_{0} j_{1} \cdots j_{k} \cdots$. Using this projection, $U_{1}$ becomes the Cantor set, and $\left\{x: \operatorname{Card}\left(S_{x}\right)=2^{u}\right\}$ becomes the set

$$
\left\{0 . j_{0} j_{1} \cdots j_{k} \cdots: \text { digit } 1 \text { appears } u \text { times in the expansion }\right\} \text {. }
$$

Let $s=\log 2 / \log 3$. Then one can check that

$$
\operatorname{dim}_{H}\left\{x: \operatorname{Card}\left(S_{x}\right)=2^{u}\right\}=s, \mathcal{H}^{s}\left\{x: \operatorname{Card}\left(S_{x}\right)=2^{u}\right\}=\infty
$$

for any $u \in \mathbf{N}^{+}$. Meanwhile, note red lines of $[0,1]^{2}$ and red points of $[0,1]$ in Fig. 4 , we have

hence

$$
U_{3 \cdot 2^{u}} \subset\left\{\frac{q_{1}}{3^{q_{2}}}\right\}_{q_{1}, q_{2} \in \mathbf{Z}} \text { and } \operatorname{Card}\left(U_{3 \cdot 2^{u}}\right)=\aleph_{0}
$$

$$
\operatorname{dim}_{H}\left(U_{3 \cdot 2^{u}}\right)=0 \text { with } \mathcal{H}^{0}\left(U_{3 \cdot 2^{u}}\right)=\infty .
$$

The paper is organized as follows. In Section 2, we provide the preliminaries, including graph-directed construction [22], multi dynamical systems for sections of self-similar sets [33] and counting formula of sections [20]. Section 3 is devoted to Theorem 1. In Section 4, we will prove Theorems 2 and 3 on $U_{r}$ with $r \geq 2$. We also give some remarks in Section 5 .

\section{Preliminaries}

Note that

$$
\prod_{i=1}^{l} K_{i}=\bigcup_{\mathbf{i} \in \prod_{i=1}^{l} \mathcal{A}_{i}} \frac{\prod_{i=1}^{l} K_{i}+\mathbf{i}}{n}
$$

For notational convenience, we write $S_{\mathbf{i}}(x)=\frac{x+\mathbf{i}}{n}$ and $S_{\mathbf{i}_{1} \cdots \mathbf{i}_{k}}=S_{\mathbf{i}_{1}} \circ \cdots \circ S_{\mathbf{i}_{k}}$. The cube $S_{\mathbf{i}_{1} \cdots \mathbf{i}_{k}}\left([0,1]^{l}\right)$ with sidelength $n^{-k}$ is said to be a basic cube of rank $k$. Denote the hyperplane

$$
H_{x}=\left\{\mathbf{y} \in \mathbf{R}^{l}:(\mathbf{m}, \mathbf{y})=x\right\} .
$$


Lemma 1. Suppose the covering condition holds for $\prod_{i=1}^{l} K_{i}$ with respect to $\mathbf{m}$. Then $H_{x}$ intersects $S_{\mathbf{i}_{1} \cdots \mathbf{i}_{k}}\left([0,1]^{l}\right)$ if and only it intersects $S_{\mathbf{i}_{1} \cdots \mathbf{i}_{k}}\left(\prod_{i=1}^{l} K_{i}\right)$.

2.1. Graph-directed construction. Recall the graph-directed construction introduced by Mauldin and Williams [22]. Given a directed graph $G=(V, E)$, suppose $\left\{H_{i}\right\}_{i}$ are its strongly connected components and $\mathcal{N}=\left(b_{v, v^{\prime}}\right)_{v, v^{\prime} \in V}$ is the matrix respect to the graph, i.e., $b_{v, v^{\prime}}$ is number of edges from $v$ to $v^{\prime}$. Given a directed edge $e$, we equip a linear mapping $g_{e}: \mathbf{R} \rightarrow \mathbf{R}$ with contracting ratio $n^{-1}$ where $n^{-1}$ is also the contracting ratio in (2.1). By the classical result of [22], there is a unique family of compact subsets $\left\{K_{v}\right\}_{v \in V} \subset \mathbf{R}$ such that

$$
K_{v}=\bigcup_{v^{\prime} \in V} \bigcup_{e \in \mathcal{E}\left(v, v^{\prime}\right)} g_{e}\left(K_{v^{\prime}}\right) \text {, }
$$

where $\mathcal{E}\left(v, v^{\prime}\right)$ denotes the set of directed edges from $v$ to $v^{\prime}$. We say that the open set condition holds, if there are non-empty open sets $\left\{U_{v}\right\}_{v \in V}$ of $\mathbf{R}$ such that

$$
\bigcup_{v^{\prime} \in V} \bigcup_{e \in \mathcal{E}\left(v, v^{\prime}\right)} g_{e}\left(U_{v^{\prime}}\right) \subset U_{v}
$$

and the left hand of the above formula is a disjoint union for each $v \in V$. By the results in [22], we have the following

Lemma 2. Suppose the open set condition holds. Then

(1) $\operatorname{dim}_{H}\left(\bigcup_{v \in V} K_{v}\right)=\frac{\log \rho(\mathcal{N})}{\log n}=s$;

(2) Suppose $s>0$, then $\mathcal{H}^{s}\left(\bigcup_{v \in V} K_{v}\right)>0$;

(3) Suppose $s>0$, then $\mathcal{H}^{s}\left(\bigcup_{v \in V} K_{v}\right)=\infty$ if and only if there are two different strongly connected components $H$ and $H^{\prime}$ such that $\rho(H)=\rho\left(H^{\prime}\right)=\rho(\mathcal{N})$ and $H \prec H^{\prime}$.

(4) Suppose $v \in H_{i}$, then

$$
\operatorname{dim}_{H} K_{v}=\max \left\{\frac{\log \rho\left(H_{j}\right)}{\log n}: H_{i} \prec H_{j}\right\} .
$$

Moreover, if $d=\operatorname{dim}_{H} K_{v}>0$, then $\mathcal{H}^{d}\left(K_{v}\right)>0$.

For example, let the symbolic system

$$
X=\left\{\bar{v}=v_{i_{0}} v_{i_{1}} \cdots v_{i_{k}} \cdots: \text { the infinite sequence } \bar{v} \text { is admissible }\right\}
$$

and $X_{v}$ the collection of infinite admissible sequences starting from $v$. There is a metric on $X$ defined by

$$
\mathrm{d}\left(v_{i_{0}} \cdots v_{i_{k}} v_{i_{k+1}} \cdots, v_{i_{0}} \cdots v_{i_{k}} v_{i_{k+1}^{\prime}} \cdots\right)=n^{-k} \text { if } v_{i_{k+1}} \neq v_{i_{k+1}^{\prime}} .
$$

As in Lemma 2, by results of [22], we conclude that if $s=\frac{\log \rho(\mathcal{N})}{\log n}>0$ then $\operatorname{dim}_{H}(X)=$ $s$ and $\mathcal{H}^{s}(X)>0$ and

$$
\operatorname{dim}_{H} X_{v}=\max \left\{\frac{\log \rho\left(H_{j}\right)}{\log n}: H_{i} \prec H_{j}\right\} \text { where } v \in H_{i} .
$$

We also have the following

Claim 1. If $\operatorname{dim}_{H} X_{v}=0$, then $X_{v}$ is countable.

Proof. It suffices to show for strongly connected component $H_{j}$ with $\rho\left(H_{j}\right) \leq 1$ the collection of admissible infinite sequences with letters in $H_{j}$ is a finite set. Suppose $\gamma$ is a Perron-Frobenius eigenvector $\gamma$ of the matrix $\mathcal{N}_{j}$ w.r.t. $H_{j}$ such that $\|\gamma\|_{1}=1$, and $\mathcal{D}_{k}$ denotes the set of admissible sequences of length $k$ such that every letter 
lies in $H_{j}$. Using the irreducibility, there exists a constant $\varsigma>0$ such that for all $k$, $\operatorname{Card}\left(\mathcal{D}_{k}\right) \leq\left\|\mathcal{N}_{j}^{k}(1, \cdots, 1)^{T}\right\|_{1} \leq \varsigma\left\|\mathcal{N}_{j}^{k} \gamma\right\|_{1}=\varsigma\left\|\rho\left(H_{j}\right)^{k} \gamma\right\|_{1} \leq \varsigma$.

2.2. Multi dynamical system. We will construct a multi dynamical system including many expanding maps from integer intervals to $\left[m_{*}, m^{*}\right]$. In fact, for every integer interval $I: n^{-1}[u, u+1] \subset\left[m_{*}, m^{*}\right]$, we equip the interval with several expanding maps with factor $n$ as follows. Suppose $I$ is of type $t$ with $u-(\mathbf{m}, \mathbf{i})=t$, we let $f_{\mathrm{i}}: I \rightarrow J_{t}=[t, t+1]$ denote the corresponding linear surjection in the form

$$
f_{\mathbf{i}}(x)=n x-u+t: I \rightarrow J_{t} .
$$

Then the multi dynamical system consists of $\left\{f_{\mathbf{i}}\right\}_{\mathbf{i} \in \prod_{i=1}^{l} \mathcal{A}_{i}}$.

The next lemma reveals the connection between the multi dynamical system and intersections of hyperplanes with basic cubes.

Lemma 3. If $x \in I=n^{-1}[u, u+1](u \in \mathbf{Z})$ and $\mathbf{i} \in \prod_{i=1}^{l} \mathcal{A}_{i}$ such that $I \subset$ $\left(\mathbf{m}, \mathbf{c}_{\mathbf{i}}\right)$, then

$$
H_{x} \bigcap S_{\mathbf{i}}\left([0,1]^{l}\right)=S_{\mathbf{i}}\left(H_{f_{\mathbf{i}}(x)} \cap[0,1]^{l}\right) .
$$

Using (2.2) again and again, we obtain then

Lemma 4. If $x \in\left(\mathbf{m}, \mathbf{c}_{\mathbf{i}_{1}}\right)$ and $\left(f_{\mathbf{i}_{j}} \circ \cdots \circ f_{\mathbf{i}_{1}}\right)(x) \in\left(\mathbf{m}, \mathbf{c}_{\mathbf{i}_{j+1}}\right)$ for all $1 \leq j \leq k-1$,

$$
H_{x} \bigcap S_{\mathbf{i}_{1} \mathbf{i}_{2} \cdots \mathbf{i}_{k}}\left([0,1]^{l}\right)=S_{\mathbf{i}_{1} \mathbf{i}_{2} \cdots \mathbf{i}_{k}}\left(H_{\left(f_{\mathbf{i}_{k}} \circ \cdots \circ f_{\mathbf{i}_{1}}\right)(x)} \cap[0,1]^{l}\right)
$$

When $I \in \Xi$, since there is only one $\mathbf{i} \in \prod_{i=1}^{l} \mathcal{A}_{i}$ such that $I \subset\left(\mathbf{m}, \mathbf{c}_{\mathbf{i}}\right)$ and thus the type $t$ is uniquely determined by $I$, we can write $f_{\mathbf{i}}$ to be $f_{I}$ for convenience.

If there is an admissible sequence $I_{i_{0}} I_{i_{1}} \cdots I_{i_{k-1}} I_{i_{k}} \cdots$ of $G_{\Xi}$ such that

$$
x \in \operatorname{int}\left(I_{i_{0}}\right) \text { and } g_{k}(x)=\left(f_{I_{i_{k-1}}} \circ \cdots \circ f_{I_{i_{0}}}\right)(x) \in \operatorname{int}\left(I_{i_{k+1}}\right) \text { for all } k \text {, }
$$

then we say that $x \in\left[m_{*}, m^{*}\right]$ has an infinite coding $I_{i_{0}} I_{i_{1}} \cdots I_{i_{k-1}} I_{i_{k}} \cdots$ of $G_{\Xi}$. By Lemma 4 we have

Lemma 5. Let $g_{k}(x)=f_{I_{i_{k-1}}} \circ \cdots \circ f_{I_{i_{0}}}(x)$ be defined as above. Then

$$
H_{x} \bigcap S_{\mathbf{i}_{1} \mathbf{i}_{2} \cdots \mathbf{i}_{k}}\left([0,1]^{l}\right)=S_{\mathbf{i}_{1} \mathbf{i}_{2} \cdots \mathbf{i}_{k}}\left(H_{g_{k}(x)} \cap[0,1]^{l}\right) .
$$

Let

$$
\Lambda=\left\{x \in\left[m_{*}, m^{*}\right]: x \text { has an infinite coding in } \Xi\right\} .
$$

On the other hand, given an admissible sequence $I_{i_{0}} I_{i_{1}} \cdots I_{i_{k-1}} I_{i_{k}} \cdots$ in $G_{\Xi}$, by the theorem of nested interval, there exists a unique $x \in\left[m_{*}, m^{*}\right]$ such that

$$
x \in I_{i_{0}} \text { and } g_{k}(x) \in I_{i_{k+1}} \text { for all } k \text {. }
$$

That means

$$
\begin{gathered}
\bar{\Lambda}=\left\{x \in\left[m_{*}, m^{*}\right]: I_{i_{0}} I_{i_{1}} \cdots I_{i_{k-1}} I_{i_{k}} \cdots \text { is admissible in } G_{\Xi}\right. \text { such that } \\
\left.x \in I_{i_{0}} \text { and } g_{k}(x) \in I_{i_{k+1}} \text { for all } k\right\}
\end{gathered}
$$

and $\bar{\Lambda} \backslash \Lambda \subset\left\{\frac{q_{1}}{n^{q_{2}}}\right\}_{q_{1}, q_{2} \in \mathbf{Z}}$.

We will consider a graph-directed construction induced by $\Xi$. Suppose $I_{1}, I_{2} \in \Xi$ with $I_{1} \rightarrow I_{2}$, the mapping

$$
g_{\left(I_{1}, I_{2}\right)}=\left.\left(f_{I_{1}}\right)^{-1}\right|_{I_{2}}: I_{2} \rightarrow I_{1}
$$


with contracting ratio $n^{-1}$. Note that the open set condition holds for this graphdirected construction. By the classical result of [22], there is a unique family of compact subsets $\left\{F_{I}\right\}_{I}$ such that $F_{I} \subset I$ for all $I \in \Xi$ and

$$
F_{I}=\bigcup_{J \in \mathcal{E}(I)} g_{(I, J)}\left(F_{J}\right)
$$

where $\mathcal{E}(I)$ denotes the set of ending vertex of the edge starting from $I$. Now, we have

$$
\bar{\Lambda}=\bigcup_{I \in \Xi} F_{I} \quad \text { with } \operatorname{dim}_{H}(\bar{\Lambda})=s
$$

Moreover, if $s>0$ then $\mathcal{H}^{s}(\bar{\Lambda})>0$ under the open set condition. Note that

$$
\bar{\Lambda} \backslash \Lambda \subset\left\{\frac{q_{1}}{n^{q_{2}}}\right\}_{q_{1}, q_{2} \in \mathbf{Z}}
$$

and $\operatorname{dim}_{H}(\Lambda)=\operatorname{dim}_{H}(\bar{\Lambda})$, by Lemma 2, we have

Lemma 6. Let $s=\frac{\log \rho(M)}{\log n}$. We have

(1) $\operatorname{dim}_{H} \Lambda=s$;

(2) If $s>0$, then $\mathcal{H}^{s}(\Lambda)>0$, moreover, $\mathcal{H}^{s}(\Lambda)=\infty$ if and only if there are two strongly connected components $\Xi_{i}$ and $\Xi_{j}$ in $\Xi$ such that $\rho\left(M_{i}\right)=\rho\left(M_{j}\right)=$ $\rho(M)$ and $\Xi_{i} \prec \Xi_{j}$. In particular, if $M$ is irreducible, then $0<\mathcal{H}^{s}(\Lambda)<\infty$.

One can check the following lemma directly in terms of Lemma 2.

Lemma 7. Suppose $\omega$ is a congruent subset of $\Xi$ and $\Omega_{i}$ is the strongly connected component of $G^{*}$ containing $\omega$. Let

$A_{\omega}=\left\{z\right.$ : vector $(z+p)_{p \in \mathcal{D}(\omega)}$ has an infinite coding in $G^{*}$ starting from $\left.\omega\right\}$.

Then $d_{\omega}=\operatorname{dim}_{H} A_{\omega}=\max \left\{\frac{\log \rho\left(\Omega_{j}\right)}{\log n}: \Omega_{i} \prec \Omega_{j}\right\}$. If $d_{\omega}>0$, then $\mathcal{H}^{d_{\omega}}\left(A_{\omega}\right)>0$.

2.3. Counting formula. For $z \in[0,1)$, we let

$$
\alpha(z)=\left(\operatorname{Card}\left(S_{z+m_{*}}\right), \operatorname{Card}\left(S_{z+m_{*}+1}\right), \cdots, \operatorname{Card}\left(S_{z+m^{*}-1}\right)\right)^{T},
$$

and

$$
\sigma:[0,1) \rightarrow[0,1) \text { such that } \sigma z=n z(\bmod 1) .
$$

Let $\{x\} \in[0,1)$ denote the decimal part of $x=[x]+\{x\}$, e.g. $\{-3.4\}=0.6$.

Using these notations and matrix $T_{j}$ in Section 1 and results in [20], we have If

Lemma 8. Suppose the covering condition holds. Let $x \in\left[m_{*}, m^{*}\right] \backslash\left\{\frac{q_{1}}{n^{q_{2}}}\right\}_{q_{1}, q_{2} \in \mathbf{Z}}$.

$$
x=i+n^{-1} j_{1}+n^{-2} j_{2}+\cdots+n^{-k} j_{k}+\cdots,
$$

i.e., $x-i$ has $n$-adic expansion $0 . j_{1} j_{2} \cdots j_{k} \cdots$, then $\left\|e_{i} T_{j_{1}} \cdots T_{j_{k}}\right\|_{1}$ is the number of basic cubes of rank $k$ intersecting the hyperplane $H_{x}$. Moreover, if the strong separation condition holds for each $K_{i}$, then

$$
\operatorname{Card}\left(S_{x}\right)=e_{i} T_{j_{1}} \cdots T_{j_{k}} \alpha\left(\sigma^{k}\{x\}\right)=\left\|e_{i} T_{j_{1}} \cdots T_{j_{k}}\right\|_{1} .
$$




\section{Dimension of set with unique solution}

Proof of Theorem 1. (1) We can show that $\operatorname{dim}_{H} U_{1} \geq s$. By Lemma 6 , we only need to verify that

$$
\Lambda \backslash\left\{\frac{q_{1}}{n^{q_{2}}}\right\}_{q_{1}, q_{2} \in \mathbf{Z}} \subset U_{1} .
$$

In fact, suppose $x \in \Lambda \backslash\left\{\frac{q_{1}}{n^{q_{2}}}\right\}_{q_{1}, q_{2} \in \mathbf{Z}}$ has an infinite coding $I_{i_{1}} \cdots I_{i_{k}} \cdots$ in $G_{\Xi}$, then $g_{k}(x) \in \operatorname{int}\left(I_{i_{k+1}}\right)$ for all $k$ as in (2.3)-(2.4). From the definition of coding and (2.2)(2.4), we obtain a family of nested cubes $\left\{Q_{k}\right\}_{k}$ such that $Q_{k}$ is a basic cube of rank $k$ and $\left(\prod_{i=1}^{l} K_{i}\right) \cap H_{x} \subset Q_{k}$ for all $k$, which implies that the intersection $\left(\prod_{i=1}^{l} K_{i}\right) \cap H_{x}$ is a singleton. Hence (3.1) follows. It follows from (3.1) and Lemma 6 that

$$
\mathcal{H}^{s}\left(U_{1}\right) \geq \mathcal{H}^{s}(\Lambda)>0 \quad \text { if } s>0 .
$$

If $s=0$, for getting $\mathcal{H}^{s}\left(U_{1}\right)>0$, we only need to show that $U_{1}$ is non-empty. In fact, we find $\operatorname{Card}\left(S_{x}\right)=1$ for $x=\sum_{m_{i}<0} m_{i}\left(\min _{x_{i} \in K_{i}} x_{i}\right)+\sum_{m_{i}>0} m_{i}\left(\max _{x_{i} \in K_{i}} x_{i}\right)$ which implies $U_{1} \neq \emptyset$.

(2) Suppose the covering condition holds. Assume that $x \notin\left\{\frac{q_{1}}{n^{q_{2}}}\right\}_{q_{1}, q_{2} \in \mathbf{Z}}$ with $\operatorname{Card}\left(S_{x}\right)=1$ and $\mathbf{y} \in \prod_{i=1}^{l} K_{i}$ is the unique solution such that $(\mathbf{m}, \mathbf{y})=x$. Denote by $N_{k}$ the number of basic cubes with rank $k$ which contain $\mathbf{y}$. By the covering condition and Lemma 1, we obtain

$$
N_{k+1} \geq N_{k} \quad \text { for all } k .
$$

Note that $N_{k} \leq 2^{l}$ for all $k$. Therefore, there is an integer $k_{0}$ such that $N_{k_{0}}=\max _{k} N_{k}$. Fix a basic cube $S_{\mathbf{i}_{1} \cdots \mathbf{i}_{k_{0}}}\left([0,1]^{l}\right)$ of rank $k_{0}$ containing $\mathbf{y}$, by Lemmas 4 and 1 we obtain that

$$
\left(f_{\mathbf{i}_{k_{0}}} \circ \cdots \circ f_{\mathbf{i}_{1}}\right)(x) \text { has an infinite coding of } G_{\Xi}
$$

Hence

$$
U_{1} \subset\left\{x:\left(f_{\mathbf{i}_{k}} \circ \cdots \circ f_{\mathbf{i}_{1}}\right)(x) \in \Lambda \text { for some } f_{\mathbf{i}_{k}} \circ \cdots \circ f_{\mathbf{i}_{1}}\right\},
$$

Since $\left\{f_{\mathbf{i}_{k}} \circ \cdots \circ f_{\mathbf{i}_{1}}\right\}_{\mathbf{i}_{1} \cdots \mathbf{i}_{k}}$ is a countable family, we obtain that

$$
\operatorname{dim}_{H} U_{1} \leq \operatorname{dim}_{H} \Lambda .
$$

It follows from (3.1)-(3.2) and $\operatorname{dim}_{H} \Lambda=s$ (Lemma 6) that

$$
\operatorname{dim}_{H} U_{1}=s
$$

under the covering condition.

(3) Suppose the covering condition and the strong separation condition hold. It follows from the strong separation condition that basic cubes of rank $k$ are pairwise disjoint. If $x \notin\left\{\frac{q_{1}}{n^{q_{2}}}\right\}_{q_{1}, q_{2} \in \mathbf{Z}}$ and $\operatorname{Card}\left(S_{x}\right)=1$ with the unique solution $\mathbf{y}$, then for each $k$ there is a unique basic cube $Q_{k}$ of rank $k$ containing $\mathbf{y}$ which means $x \in \Lambda$. Therefore

$$
\left(U_{1} \backslash \Lambda\right) \cup\left(\Lambda \backslash U_{1}\right) \subset\left\{\frac{q_{1}}{n^{q_{2}}}\right\}_{q_{1}, q_{2} \in \mathbf{Z}} .
$$

The other part of Theorem 1 follows from Lemma 6 and (3.3). 


\section{Number of solutions}

Proof of Theorem 2. Suppose

$$
x=i+n^{-1} j_{1}+n^{-2} j_{2}+\cdots+n^{-k} j_{k}+\cdots \in\left[m_{*}, m^{*}\right] \backslash\left\{\frac{q_{1}}{n^{q_{2}}}\right\}_{q_{1}, q_{2} \in \mathbf{Z}} .
$$

Let $\operatorname{Card}\left(S_{x}\right)=r$ with solution set $\left\{\mathbf{y}_{1}, \cdots, \mathbf{y}_{r}\right\}$. Denote by $N_{k}$ the number of basic cubes of rank $k$ intersecting $\left\{\mathbf{y}_{1}, \cdots, \mathbf{y}_{r}\right\}$. It follows from the covering condition that

$$
N_{k+1} \geq N_{k} \text { for all } k \text {. }
$$

Note that $N_{k} \leq r$ for all $k$ due to the strong separation condition. Then there exists an integer $k_{0}$ such that $N_{k_{0}}=r$ and thus

$$
\operatorname{Card}\left(S_{x}\right)=e_{i} T_{j_{1}} \cdots T_{j_{k_{0}}} \alpha\left(\sigma^{k_{0}}\{x\}\right)=\left\|e_{i} T_{j_{1}} \cdots T_{j_{k_{0}}}\right\|_{1}=r
$$

due to $(2.7)$ in Lemma 8. Let $\left(\beta_{m_{*}}, \cdots, \beta_{m^{*}-1}\right)=e_{i} T_{j_{1}} \cdots T_{j_{k_{0}}}$ and $\mathcal{D}=\left\{p: \beta_{p} \neq 0\right\}$. Then $\sigma^{k_{0}}\{x\}+p \in U_{1}$ for all $p \in \mathcal{D}$, that means $\sigma^{k_{0}}\{x\}+p$ has an infinite coding in $G_{\Xi}$ for all $p \in \mathcal{D}$. Hence

$$
\left\{z \in[0,1) \backslash\left\{\frac{q_{1}}{n^{q_{2}}}\right\}_{q_{1}, q_{2} \in \mathbf{Z}}:(z+p)_{p \in \mathcal{D}} \text { has an infinite coding in } G^{*}\right\} \neq \emptyset .
$$

On the other hand, if $r=\left\|e_{i} T_{j_{1}} \cdots T_{j_{k}}\right\|_{1}$ with $e_{i} T_{j_{1}} \cdots T_{j_{k}}=\left(\beta_{m_{*}}, \cdots, \beta_{m^{*}-1}\right)$ and $\mathcal{D}=\left\{p: \beta_{p} \neq 0\right\}$ satisfying

$$
\left\{z \in[0,1) \backslash\left\{\frac{q_{1}}{n^{q_{2}}}\right\}_{q_{1}, q_{2} \in \mathbf{Z}}:(z+p)_{p \in \mathcal{D}} \text { has an infinite coding in } G^{*}\right\} \neq \emptyset .
$$

Then we can construct

$$
x=\left(i+\sum_{k=1}^{k_{0}} n^{-k} j_{k}+n^{-k_{0}} z\right) \in[0,1) \backslash\left\{\frac{q_{1}}{n^{q_{2}}}\right\}_{q_{1}, q_{2} \in \mathbf{Z}}
$$

and obtain that $\operatorname{Card}\left(S_{x}\right)=r$ using (2.7) again.

Proof of Theorem 3. (1) First we will show that $\operatorname{dim}_{H} U_{r} \leq \frac{\log \rho(M)}{\log n}$. Let $\operatorname{Card}\left(S_{x}\right)=r$ with solution set $\left\{\mathbf{y}_{1}, \cdots, \mathbf{y}_{r}\right\}$. Denote by $N_{k}$ the number of basic cubes of rank $k$ intersecting $\left\{\mathbf{y}_{1}, \cdots, \mathbf{y}_{r}\right\}$. It follows from the covering condition that

$$
N_{k+1} \geq N_{k} \text { for all } k \text {. }
$$

Note that $N_{k} \leq 2^{l} r$ for all $k$. Therefore, there exists an integer $k_{0}$ such that $N_{k_{0}}=$ $\max _{k} N_{k}$. Fix a basic cube $S_{\mathbf{i}_{1} \cdots \mathbf{i}_{k_{0}}}\left([0,1]^{l}\right)$ of rank $k_{0}$ intersecting $\left\{\mathbf{y}_{1}, \cdots, \mathbf{y}_{r}\right\}$, by Lemma 4, we obtain that

$$
\left(f_{\mathbf{i}_{k_{0}}} \circ \cdots \circ f_{\mathbf{i}_{1}}\right)(x) \text { has an infinite coding of } G_{\Xi} .
$$

Hence

$$
U_{r} \subset\left\{x:\left(f_{\mathbf{i}_{k}} \circ \cdots \circ f_{\mathbf{i}_{1}}\right)(x) \in \Lambda \text { for some } f_{\mathbf{i}_{k}} \circ \cdots \circ f_{\mathbf{i}_{1}}\right\},
$$

Since $\left\{f_{\mathbf{i}_{k}} \circ \cdots \circ f_{\mathbf{i}_{1}}\right\}_{\mathbf{i}_{1} \cdots \mathbf{i}_{k}}$ is a countable family, we obtain

$$
\operatorname{dim}_{H} U_{r} \leq \operatorname{dim}_{H} \Lambda=\frac{\log \rho(M)}{\log n} .
$$

(2) We will obtain the dimension of $U_{r}$. Suppose $U_{r}$ is uncountable. Let $x=$ $i+n^{-1} j_{1}+n^{-2} j_{2}+\cdots+n^{-k} j_{k}+\cdots \in\left[m_{*}, m^{*}\right] \backslash\left\{\frac{q_{1}}{n^{q_{2}}}\right\}_{q_{1}, q_{2} \in \mathbf{Z}}$. Suppose $N_{k}$ is defined as above, by the above discussion, there exists an integer $k_{0}$ such that $N_{k_{0}}=r$ and thus

$$
\operatorname{Card}\left(S_{x}\right)=e_{i} T_{j_{1}} \cdots T_{j_{k_{0}}} \alpha\left(\sigma^{k_{0}}\{x\}\right)=\left\|e_{i} T_{j_{1}} \cdots T_{j_{k_{0}}}\right\|_{1}=r
$$


due to $(2.7)$ in Lemma 8. Let $\left(\beta_{m_{*}}, \cdots, \beta_{m^{*}-1}\right)=e_{i} T_{j_{1}} \cdots T_{j_{0}}$ and $\mathcal{D}_{x}=\left\{p: \beta_{p} \neq 0\right\}$. When $x$ is fixed, we use $\mathcal{D}$ to replace $\mathcal{D}_{x}$ for notational convenience.

Then the above formula (4.1) implies that

$$
\sigma^{k_{0}}\{x\}+p \in U_{1} \quad \text { if } p \in \mathcal{D} .
$$

Let $\sigma^{k_{0}}\{x\} \in n^{-1}\left[h_{x}, h_{x}+1\right)$. Then $\sigma^{k_{0}}\{x\}+p \in \Lambda$ has an infinite coding in $G_{\Xi}$ for any $p \in \mathcal{D}$. Let

$$
\mathcal{F}_{\mathcal{D}}=\left\{\omega_{h}: \omega_{h}=\left\{n^{-1}[n p+h, n p+h+1]\right\}_{p \in \mathcal{D}} \in \Xi \text { with } h \in[0,(n-1)] \cap \mathbf{Z}\right\} .
$$

Suppose $\omega_{h} \in \mathcal{F}_{\mathcal{D}}$ and $\Omega_{i(h, \mathcal{D})}$ is the strongly connected components containing $\omega_{h}$. If $\sigma^{k_{0}}\{y\}=z \in n^{-1}[h, h+1)$, using the formula in Lemma 8 we have

$$
\operatorname{Card}\left(S_{y}\right)=e_{i} T_{j_{1}} \cdots T_{j_{k_{0}}} \alpha(z)=\left(\beta_{m_{*}}, \cdots, \beta_{m^{*}-1}\right) \alpha(z)=r
$$

i.e.,

$$
\left\{y=i+\sum_{k=1}^{k_{0}} n^{-k} j_{k}+n^{-k_{0}} z: z \in \Lambda \cap[0,1) \text { whenever } p \in \mathcal{D}\right\} \subset U_{r} .
$$

In fact, $\sigma^{k_{0}}\{y\}+p \in \Lambda$ for all $p \in \mathcal{D}$ implies that $(z+p)_{p \in \mathcal{D}}$ has an infinite coding in $G^{*}$ starting from $\omega_{h}$, i.e., $z \in A_{\omega_{h}}$, where $A_{\omega_{h}}$ is defined in Lemma 7. Using Lemma 7 we have

$$
\operatorname{dim}_{H} U_{r} \geq \operatorname{dim}_{H} A_{\omega_{h}}=\max \left\{\frac{\log \rho\left(\Omega_{j}\right)}{\log n}: \Omega_{i(h, \mathcal{D})} \prec \Omega_{j}\right\}
$$

for any $\omega_{h} \in \mathcal{F}_{\mathcal{D}}=\mathcal{F}_{\mathcal{D}_{x}}$. In particular, for $d^{\prime}=\max \left\{\frac{\log \rho\left(\Omega_{j}\right)}{\log n}: \Omega_{i(h, \mathcal{D})} \prec \Omega_{j}\right\}$, using Lemma 7 again we have

$$
\mathcal{H}^{d^{\prime}}\left(U_{r}\right) \geq\left(n^{-k_{0}}\right)^{d^{\prime}} \mathcal{H}^{d^{\prime}}\left(A_{\omega_{h}}\right)>0 \quad \text { if } d^{\prime}>0 .
$$

Let $\mathcal{G}=\left\{\mathcal{D}_{x}: \operatorname{Card}\left(S_{x}\right)=r\right\}$. The above discussion shows that

$$
\text { if } x \in U_{r} \backslash\left\{\frac{q_{1}}{n^{q_{2}}}\right\}_{q_{1}, q_{2} \in \mathbf{Z}}, \quad \text { then } \sigma^{k_{0}}\{x\} \in A_{\omega_{h_{x}}} \text { for some } k_{0} \text {. }
$$

Hence

$$
\begin{aligned}
\operatorname{dim}_{H}\left(U_{r}\right) & =\operatorname{dim}_{H}\left(U_{r} \backslash\left\{\frac{q_{1}}{n^{q_{2}}}\right\}_{q_{1}, q_{2} \in \mathbf{Z}}\right) \leq \max _{\mathcal{D} \in \mathcal{G}} \operatorname{dim}_{H}\left(\bigcup_{\omega \in \mathcal{F}_{\mathcal{D}}} A_{\omega}\right) \\
& \leq \max _{\mathcal{D} \in \mathcal{G}} \max _{\omega_{h} \in \mathcal{F}_{\mathcal{D}}} \max \left\{\frac{\log \rho\left(\Omega_{j}\right)}{\log n}: \Omega_{i(h, \mathcal{D})} \prec \Omega_{j}\right\} .
\end{aligned}
$$

i.e.,

$$
d_{r}=\operatorname{dim}_{H} U_{r}=\max _{\mathcal{D} \in \mathcal{G}} \max _{\omega_{h} \in \mathcal{F}_{\mathcal{D}}} \max \left\{\frac{\log \rho\left(\Omega_{j}\right)}{\log n}: \Omega_{i(h, \mathcal{D})} \prec \Omega_{j}\right\} .
$$

By (4.2) and (4.3), we obtain that

$$
\mathcal{H}^{d_{r}}\left(U_{r}\right)>0 \text { if } d_{r}>0 .
$$

It suffices to show that $d_{r}>0$. In fact, by the above discussion we have

$$
\mathcal{U}_{r} \subset \bigcup_{\mathcal{D} \in \mathcal{G}} \bigcup_{\omega_{h} \in \mathcal{F}_{\mathcal{D}}} \bigcup_{\left(i, j_{1}, \cdots j_{k_{0}}\right)}\left\{y=i+\sum_{k=1}^{k_{0}} n^{-k} j_{k}+n^{-k_{0}} z: z \in A_{\omega_{h}}\right\} \text {, }
$$

where $\mathcal{U}_{r}=U_{r} \backslash\left\{\frac{q_{1}}{n^{q_{2}}}\right\}_{q_{1}, q_{2} \in \mathbf{Z}}$. Note that

$$
d_{r}=\max _{\mathcal{D} \in \mathcal{G}} \max _{\omega_{h} \in \mathcal{F}_{\mathcal{D}}} \operatorname{dim}_{H}\left(A_{\omega_{h}}\right) .
$$

If $d_{r}=0$, then $\operatorname{dim}_{H}\left(A_{\omega_{h}}\right)=0$ for all $\omega_{h}$ which implies that $A_{\omega_{h}}$ is countable for all $\omega_{h}$ due to Claim 1. Using (4.4) we obtain that $U_{r}$ is countable, this is a contradiction. 
(3) Now we will obtain the infinity of Hausdorff measure $\mathcal{H}^{d_{r}}\left(U_{r}\right)$ when

$$
\mathcal{U}_{r}=U_{r} \backslash\left\{\frac{q_{1}}{n^{q_{2}}}\right\}_{q_{1}, q_{2} \in \mathbf{Z}} \neq \emptyset \quad \text { with } r \geq 2
$$

and $\left[m_{*}, m^{*}\right]$ is dominated by $\Xi$ for $d_{r}$. Take $x \in \mathcal{U}_{r}$ and $\omega_{h} \in \mathcal{F}_{\mathcal{D}_{x}}$ such that

$$
\max \left\{\frac{\log \rho\left(\Omega_{j}\right)}{\log n}: \Omega_{i(h, \mathcal{D})} \prec \Omega_{j}\right\}=d_{r},
$$

where $\Omega_{i(h, \mathcal{D})}$ is the strongly connected component containing $\omega_{h}$. It follows from Lemma 7 that

$$
\mathcal{H}^{d_{r}}\left(A_{\omega_{h}}\right)>0
$$

Since $\left[m_{*}, m^{*}\right]$ is dominated by $\Xi$ for $d_{r}$, we note that for $x=i+\sum_{k=1}^{k_{0}} n^{-k} j_{k}+\cdots \in J_{i}$ as above, we take a strongly connected component $\Xi_{j}$ of $G_{\Xi}$ with $\frac{\log \rho\left(\Xi_{j}\right)}{\log n} \geq d_{r}$ such that there is a path $\mathbf{P}^{\prime}$ in $G_{\Xi}$ from some $I \in \Xi_{j}$ to an integer interval $I^{\prime} \in \Xi$ of type $i$. Let

$$
\mathcal{C}_{k}=\left\{\mathbf{P}: \mathbf{P} \text { is a path in } \Xi_{j} \text { ending at } I \text { and }|\mathbf{P}|=k\right\},
$$

where $|\mathbf{P}|$ denotes the length of the path. Then we obtain a family $\left\{\mathcal{B}_{k}\right\}_{k}$ of pairwise disjoint subsets of $U_{r}$ as follows

$$
\mathcal{B}_{k}=\left\{\mathbf{P P}^{\prime}\left(i+\sum_{k=1}^{k_{0}} n^{-k} j_{k}+n^{-k_{0}} z\right): \mathbf{P} \in \mathcal{C}_{k} \text { and } z \in A_{\omega_{h}}\right\} \quad \text { for } k=1,2, \cdots,
$$

where the coding $\mathbf{P} \mathbf{P}^{\prime} x_{1}$ denotes a real number which is turned to be $x_{1} \in[t, t+1]$ through a series of expanding maps with respect to the path $\mathbf{P P}^{\prime}$ in $\Xi$ and the expanding map according to $I^{\prime} \longmapsto J_{i}$. Hence

$$
\mathcal{H}^{d_{r}}\left(\mathcal{B}_{k}\right) \geq \operatorname{Card}\left(\mathcal{C}_{k}\right)\left(n^{-\left(k+k_{0}\right) d_{r}} \mathcal{H}^{d_{r}}\left(A_{\omega_{h}}\right)\right),
$$

and there is a constant $c>0$ such that

$$
\operatorname{Card}\left(\mathcal{C}_{k}\right) \geq c \rho\left(\Xi_{j}\right)^{k} \geq c n^{d_{r} k} .
$$

Here we can check $\operatorname{Card}\left(\mathcal{C}_{k}\right) \geq c \rho\left(\Xi_{j}\right)^{k}$ in (4.6), because by the irreducibility we can take a Perron-Frobenius eigenvector $\gamma$ of the matrix $M_{j}$ w.r.t. $\Xi_{j}$ such that $\|\gamma\|_{1}=1$, and thus

$$
\operatorname{Card}\left(\mathcal{C}_{k}\right) \geq c\left\|M_{j}^{k}(1, \cdots, 1)^{T}\right\|_{1} \geq c\left\|M_{j}^{k} \gamma\right\|_{1}=c\left\|\rho\left(\Xi_{j}\right)^{k} \gamma\right\|_{1} \geq c \rho\left(\Xi_{j}\right)^{k} .
$$

It follows from (4.5) and (4.6) that there is a constant $c^{\prime}>0$ such that

$$
\mathcal{H}^{d_{r}}\left(\mathcal{B}_{k}\right) \geq c^{\prime} \text { for all } k \text {. }
$$

Hence

$$
\mathcal{H}^{d_{r}}\left(U_{r}\right) \geq \mathcal{H}^{d_{r}}\left(\bigcup_{k} \mathcal{B}_{k}\right) \geq \sum_{k} \mathcal{H}^{d_{r}}\left(\mathcal{B}_{k}\right)=+\infty \text {. }
$$

\section{Final remarks}

From the slicing point of view, our results can be restated. For instance, the results concerning with the set $U_{r}=\left\{x: \operatorname{Card}\left(S_{x}\right)=r\right\}(r<\infty)$ in Theorems 1, 2 and 3 are still correct for the following systems: let $K \subset[0,1]^{l}$ be a self-similar set with IFS $\left\{\mathbf{y} \rightarrow n^{-1}(\mathbf{y}+\mathbf{a})\right\}_{\mathbf{a} \in \mathcal{A}}$, where $\mathcal{A} \subset\{0,1, \cdots,(n-1)\}^{l}$. We do not need to modify the definitions of the directed graphs and the proofs.

The covering condition is essential in this paper. Without this condition, it is much more difficult to analyze the set $U_{r}$. We shall discuss this case in another paper. Our main ideas may be implemented in the setting of some overlapping selfsimilar sets. Nevertheless, the discussion is more complicated. We consider only the 
addition or subtraction on the Cantor sets. It is natural to consider similar problems for the multiplication or division on self-similar sets, for instance, the set of points with unique representations.

\section{References}

[1] Akiyama, S., and K. Scheicher: From number systems to shift radix systems. - Nihonkai Math. J. 16:2, 2005, 95-106.

[2] Athreya, J. S., B. Reznick, and J. T. Tyson: Cantor set arithmetic. - Amer. Math. Monthly (to appear).

[3] BAKeR, S.: Generalized golden ratios over integer alphabets. - Integers, 14:A15, 2014, 28.

[4] BÁRÁNy, B., and M. Rams: Dimension of slices of Sierpinski-like carpets. - J. Fractal Geom. $1: 3,2014,273-294$.

[5] Barrionuevo, J., R. M. Burton, K. Dajani, and C. KraAikamp: Ergodic properties of generalized Lüroth series. - Acta Arith. 74:4, 1996, 311-327.

[6] Dajani, K., and M. DE VRIES: Measures of maximal entropy for random $\beta$-expansions. - J. Eur. Math. Soc. (JEMS) 7:1, 2005, 51-68.

[7] Dajani, K., and M. DE VRIEs: Invariant densities for random $\beta$-expansions. - J. Eur. Math. Soc. (JEMS) 9:1, 2007, 157-176.

[8] Dajani, K., K. Jiang, D. Kong, and W. Li: Multiple codings for self-similar sets with overlaps. - arXiv:1603.09304, 2016.

[9] Dajani, K., K. Jiang, D. Kong, and W. Li: Multiple expansions of real numbers with digits set $\{0,1, q\}$. Math. Z. (to appear).

[10] Dajani, K., and C. KraAikamp: - Ergodic theory of numbers. - Carus Math. Monogr. 29, Math. Assoc. America, Washington, DC, 2002.

[11] Dajani, K., and M. Oomen: Random $N$-continued fraction expansions. - J. Approx. Theory 227, 2018, 1-26.

[12] De Vries, M., and V. Komornik: Unique expansions of real numbers. - Adv. Math. 221:2, 2009, 390-427.

[13] Erdốs, P., M. Horváth, and I. Joó: On the uniqueness of the expansions $1=\sum q^{-n_{i}}$. Acta Math. Hungar. 58:3-4, 1991, 333-342.

[14] Glendinning, P., and N. Sidorov: Unique representations of real numbers in non-integer bases. - Math. Res. Lett. 8:4, 2001, 535-543.

[15] Hall, M., JR.: On the sum and product of continued fractions. - Ann. of Math. (2) 48, 1947, 966-993.

[16] Hochman, M., and P. Shmerkin: Local entropy averages and projections of fractal measures. - Ann. of Math. (2) 175:3, 2012, 1001-1059.

[17] JiAng, K., and K. Dajani: Subshifts of finite type and self-similar sets. - Nonlinearity 30:2, 2017, 659-686.

[18] Komornik, V.: Expansions in noninteger bases. - Integers 11B:A9, 2011, 30.

[19] Komornik, V., D. Kong, and W. Li: Hausdorff dimension of univoque sets and devil's staircase. - Adv. Math. 305, 2017, 165-196.

[20] LiU, Q., L. XI, and Y. ZhaO: Dimensions of intersection of the Sierpinski carpet with lines of rational slopes. - Proc. Edinb. Math. Soc. (2) 50, 2007, 411-428.

[21] Manning, A., and K. Simon: Dimension of slices through the Sierpinski carpet. - Trans. Amer. Math. Soc. 365:1, 2013, 213-250.

[22] Mauldin, R. M., and S. C. Williams: Hausdorff dimension in graph directed constructions. - Trans. Amer. Math. Soc. 309:1-2, 1988, 811-839. 
[23] Parry, W.: On the $\beta$-expansions of real numbers. - Acta Math. Acad. Sci. Hungar. 11, 1960, 401-416.

[24] Peres, Y., and P. Shmerkin: Resonance between Cantor sets. - Ergodic Theory Dynam. Systems 29:1, 2009, 201-221.

[25] RÉNYI, A.: Representations for real numbers and their ergodic properties. - Acta Math. Acad. Sci. Hungar. 8, 1957, 477-493.

[26] Sidorov, N.: Almost every number has a continuum of $\beta$-expansions. - Amer. Math. Monthly $110: 9,2003,838-842$.

[27] Sidorov, N.: Universal $\beta$-expansions. - Period. Math. Hungar. 47:1-2, 2003, 221-231.

[28] Sidorov, N.: Expansions in non-integer bases: lower, middle and top orders. - J. Number Theory 129:4, 2009, 741-754.

[29] Steinhaus, H.: Mowa Własność Mnogości Cantora. - Wector 1-3, 1985.

[30] Steve, A.: Cantor sets and numbers with restricted partial quotients. - Trans. Amer. Math. Soc. $352: 1,2000,133-170$.

[31] Tian, L., J. Gu, Q. Ye, L.-F. XI, and K. Jiang: Multiplication on self-similar sets with overlaps. - arXiv:1807.05368, 2018.

[32] Wen, Z., and L. Xı: On the dimensions of sections for the graph-directed sets. - Ann. Acad. Sci. Fenn. Math. 35, 2010, 515-535.

[33] XI, L.: Rectifiable sub-surfaces of self-similar sets with cubic patterns. - Manuscript (submitted), 2018.

Received 29 August 2018 • Accepted 27 February 2019 